The Astrophysical JouRnal, 480:383-394, 1997 May 1

(C) 1997. The American Astronomical Society. All rights reserved. Printed in U.S.A.

\title{
WHOLE EARTH TELESCOPE OBSERVATIONS OF THE HELIUM INTERACTING BINARY PG $1346+082$ (CR BOOTIS)
}

\author{
J. L. Provencal ${ }^{1,2}$ D. E. Winget, ${ }^{1}$ R. E. Nather, ${ }^{1}$ E. L. Robinson, ${ }^{1}$ J. C. Clemens,,${ }^{3,4}$ \\ P. A. Bradley, ${ }^{5}$ C. F. Claver, ${ }^{6}$ S. J. Kleinman, ${ }^{7}$ A. D. Grauer, ${ }^{8,9}$ B. P. Hine, ${ }^{10,11}$ \\ L. Ferrario, ${ }^{12}$ D. O'Donoghue ${ }^{13}$ B. Warner ${ }^{13}$ G. Vauclair, ${ }^{14}$ M. CheVreton, ${ }^{15}$ \\ S. O. KePleR ${ }^{16,17}$ M. A. WOOD, ${ }^{18}$ AND G. W. HeNRY ${ }^{17,19}$ \\ Received 1996 September 30; accepted 1996 November 19
}

\begin{abstract}
We present our analysis of $240 \mathrm{hr}$ of white-light, high-speed photometry of the dwarf nova-like helium variable PG $1346+082$ (CR Boo). We identify two frequencies in the low-state power spectrum, at $679.670 \pm 0.004 \mu \mathrm{Hz}$ and $669.887 \pm 0.008 \mu \mathrm{Hz}$. The $679.670 \mu \mathrm{Hz}$ variation is coherent over at least a 2 week time span, the first demonstration of a phase-coherent photometric variation in any dwarf novalike interacting binary white dwarf system. The high-state power spectrum contains a complex fundamental with a frequency similar, but not identical, to the low-state spectrum, and a series of harmonics not detected in low state. We also uncover an unexpected dependence of the high-frequency power's amplitude and frequency structure on overall system magnitude. We discuss these findings in light of the general AM CVn system model, particularly the implications of the high-order harmonics on future studies of disk structure, mass transfer, and disk viscosity.
\end{abstract}

Subject headings: stars: individual (PG 1346+082) — stars: oscillations - white dwarfs

\section{INTRODUCTION}

PG $1346+082$ (CR Boo) is a member of a special subset of short-period binary systems, the interacting binary white dwarfs (IBWDs). IBWDs, also known as AM CVn stars, after the prototype, contain two white dwarfs of extreme mass ratio in close proximity. The secondary, with an estimated mass ranging from 0.02 to $0.09 M_{\odot}$, fills its Roche lobe and transfers material to the much more massive primary via an accretion disk (Faulkner, Flannery, \& Warner 1972). IBWDs are fascinating laboratories of accretion dynamics. Recent spectroscopic evidence argues that the prototype, AM CVn, contains a stable accretion disk with a $13.4 \mathrm{hr}$ precession period (Patterson, Halpern, \&

\footnotetext{
${ }^{1}$ Department of Astronomy and McDonald Observatory, University of Texas, Austin, TX 78712; jlp@chopin.udel.edu.

${ }^{2}$ Department of Physics and Astronomy, University of Delaware, Newark, DE 19716.

${ }^{3}$ Division of Physics, Mathematics, \& Astronomy, California Institute of Technology, Pasadena, CA 91125.

${ }^{4}$ Hubble Fellow.

5 XTA MS B220, Los Alamos National Laboratory, Los Alamos, NM 87545.

${ }^{6}$ Kitt Peak National Observatory, NOAO, Tucson, AZ 85726-6732.

${ }^{7}$ Big Bear Solar Observatory, Big Bear City, CA.

${ }^{8}$ Department of Physics and Astronomy, University of Arkansas, 2801 South University Avenue, Little Rock, AR 72204.

${ }^{9}$ Guest Observer, Siding Spring Observatory, Australia.

${ }^{10}$ NASA Ames Research Center, M.S. 244-4, Moffett Field, CA 94035.

${ }_{11}$ Visiting Astronomer, Institute for Astronomy, Honolulu, HI.

${ }^{12}$ Australian National University, Canberra, Australia.

${ }^{13}$ Department of Astronomy, University of Cape Town, Rondebosch 7700 , South Africa.

${ }^{14}$ Observatoire Midi-Pyrenees, 14 Avenue E. Belin, 31400 Toulouse, France.

${ }_{15}$ Observatoire de Paris-Meudon, F-92195 Meudon, Principal Cedex, France.

${ }^{16}$ Instituto de Fisica, Universidade Federal do Rio Grande do Sul, 91501-970 Porto Alegre-RS, Brazil.

${ }^{17}$ Visiting Astronomer, Cerro Tololo Inter-American Observatory.

${ }^{18}$ Department of Physics and Space Sciences, Florida Institute of Technology, 150 West University Boulevard, Melbourne, FL 32901.

${ }^{19}$ Center for Excellence in Information Systems, Tennessee State University, 330 10th Avenue North, Nashville, TN 37203.
}

Shambrook 1993). AM CVn stars also provide insight into binary star evolution, while offering an unique opportunity to peer into a stellar core remnant and directly view the by-products of nucleosynthesis (Marsh, Horne, \& Rosen 1991). The extremely low secondary mass suggests the donated material originates from a present surface, once deep within the core of the progenitor main-sequence star. Yet spectra of all AM CVn stars are helium-rich, rather than carbon-dominated, indicating that the evolutionary path traversed by these systems precludes the ignition of helium in the core of the secondary progenitor. Helium-core white dwarfs created by single-star evolution could not have yet formed in the age of the Galactic disk (Winget et al. 1987).

In addition, the AM CVn stars offer a possible channel onto the white dwarf cooling track. Recent theoretical and observational work (see Fontaine \& Wesemael 1996 for a review) argues that most, if not all, hydrogen-surface white dwarfs (DAs), which comprise $80 \%$ of the total white dwarf population, have thick $\left(\approx 10^{-4} M_{\odot}\right)$ surface layers, and comprise a fairly well-understood channel of white dwarf evolution. The situation is less clear for helium-surface white dwarfs (DBs), which comprise the remaining $20 \%$ of the population. Observational evidence suggests a range of helium layer thicknesses, which could be explained through either time-dependent diffusion (Dehner \& Kawaler 1995; Provencal et al. 1996) or multiple sources for DBs, such as the subdwarfs, the DAO stars, and the AM CVn systems (Shipman 1996). Evidence abounds for an evolutionary trend between orbital separation and accretion rate among the six AM CVn objects (Table 1) (Provencal 1994). The mass transfer will not stop until the secondary is completely absorbed, leaving a solitary DB (Nather, Robinson, \& Stover 1981), perhaps providing a significant fraction of the field DBs we observe today.

The Palomar Green survey (Green, Schmidt, \& Liebert 1986) object PG 1346+082 (CR Boo), one of three dwarf nova-like AM CVn systems, undergoes outbursts ranging from a low-state (minimum light) magnitude of $\approx 17.4\left(m_{b}\right)$ 